Clío América/ Vol. 14, No. 28 - 2020 / 493 - 503

http://dx.doi.org/10.21676/23897848.3975

\title{
Comercio internacional entre Alianza del Pacífico y ANSEA: un análisis comparativo
}

\author{
International trade between the Pacific Alliance and ASEAN: a comparative analysis
}

Flavio Rafael González-Ayala ${ }^{1}$ y Jorge Isaac Lechuga-Cardozo ${ }^{2}$ (i)

${ }^{1}$ Universidad Autónoma de San Luis Potosi, México.Email: flavio.gonzalez@eco.uaslp.mx

${ }^{2}$ Universidad de la Salle, Colombia. Email: jlechuga@unisalle.edu.co

Para citar este artículo: González-Ayala, F. y Lechuga-Cardozo, J. (2020). Comercio Internacional entre Alianza del Pacífico y ANSEA: un análisis comparativo. Clío América, 14(28), 493-503. http://dx.doi.org/10.21676/23897848.3975

Recibido: 24 marzo de 2020

Aceptado: 18 de agosto de 2020

Publicado en línea: noviembre 20 de 2020

Palabras clave: alianza del pacífico; ANSEA; comercio internacional; exportaciones; importaciones.

JEL: P33;

F15; P45.

Keywords: ASEAN; exports; imports; international trade; pacific alliance.

\section{RESUMEN}

El presente artículo tiene como objetivo mostrar evidencia del comportamiento de la balanza comercial, exportaciones e importaciones entre la Alianza del Pacífico - AP y la Asociación de Naciones del Sudeste Asiático - ANSEA de forma comparativa, en el período 2002 a 2018. Se definió un estudio cuantitativo de tipo correlacional, descriptivo, no experimental y transeccional. Se aceptan las hipótesis de que existen diferencias significativas entre la media de crecimiento de exportaciones, importaciones y balanza comercial de la Alianza del Pacífico, y la media de crecimiento de exportaciones, importaciones y balanza comercial de ANSEA; y se rechaza la hipótesis de que no existen diferencias significativas (iguales) entre la media de crecimiento de exportaciones e importaciones de la Alianza del Pacífico y la media de crecimiento, exportaciones e importaciones de ANSEA. Lo anterior no explica el comportamiento de la balanza de pagos ya que existen diferencias significativas entre el crecimiento de las exportaciones e importaciones de AP y ANSEA.

\section{ABSTRACT}

This article aims to show evidence of the behavior of the trade balance, exports, and imports between the Pacific Alliance PA and the Association of Southeast Asian Nations - ASEAN comparatively, in the period 2002 to 2018. A quantitative, correlational, descriptive, non-experimental and cross-sectional study was defined. The hypotheses that there are significant differences between the average growth of exports, imports and trade balance of the Pacific Alliance and the average growth of exports, imports and trade balance of ASEAN are accepted. On the contrary, the hypothesis that there are no significant (equal) differences between the average growth of exports and imports of the Pacific Alliance and the average growth of exports and imports of ASEAN is rejected. This does not explain the behavior of the balance of payments since there are significant differences between the growth of exports and imports of PA and ASEAN. 


\section{INTRODUCCIÓN}

La Alianza del Pacífico - AP se ha transformado en una plataforma crucial para el comercio internacional de sus miembros con el mundo. Su tercer objetivo es "Convertirse en una plataforma de articulación política, integración económica y comercial, y proyección al mundo, con énfasis en Asia - Pacífico" (Alianza del Pacífico, 2015).

El Asia - Pacífico es un polo de desarrollo para la economía global, con creciente protagonismo en los flujos de comercio internacional y con buenas proyecciones económicas para los próximos años. Desde los años noventa, la región ha sido un referente en materia de procesos de integración económica, teniendo como pilares la liberalización comercial, la facilitación del comercio y la cooperación económica en la región. Dicho regionalismo ha generado vínculos a través del Pacífico y hoy por hoy es la base del desarrollo de acuerdos comerciales y procesos de integración económica como la Asociación de Naciones del Sudeste Asiático - ANSEA. Tanto AP como ANSEA son dos de las iniciativas regionales más dinámicas en el contexto actual (Pérez y Uribe, 2018).

El Dr. Juan José Ramírez Bonilla en una entrevista dio una clara definición de Asia - Pacífico, comentando que su nombre es una mala traducción, ya que en inglés tiene dos connotaciones: una estrictamente geográfica, que se traduciría en español como Asia del Pacífico y allí se estarían incluyendo los países asiáticos que tienen costas sobre el Pacífico, que sería desde el Japón, Corea, China, pasando por los del Sudeste de Asia del Pacífico, Vietnam, Tailandia, Malasia, Singapur, Indonesia, Brunei Darussalam y Filipinas. La otra acepción es de carácter político y en ella se incluye a Australia y Nueva Zelanda, lo cual es resultado de la inclusión del proyecto original del Foro Económico de Asia Pacífico - APEC que no incluía a Canadá, ni Estados Unidos, ni a países Latinoamericanos, sino que era exclusivamente de los de Asia del Pacífico y Oceanía. De allí el nombre de Asia Pacific Economic Cooperation.

El tercer significado fue introducido por la ANSEA en su foro regional para denotar su área de interés, insistiendo en que Asía - Pacífico es todo el continente asiático, desde el medio oriente y la costa mediterránea hasta la costa del Pacífico, más toda la región del Pacífico, incluyendo Asia del Pacífico, Oceanía y la vertiente americana del Pacífico (Ramírez, 2018). En este sentido, la Alianza del Pacífico significa una vía para la diversificación de mercados de Asia - Pacífico, que hoy por hoy es la región comercial con mayores expectativas de crecimiento. Asimismo, contribuye a la integración regional y la apertura comercial de los países que la integran.

Ahora bien, el presente documento consiste en un estudio comparativo de la balanza comercial entre las plataformas AP y ANSEA, utilizando estadísticas descriptivas y de correlación. El tema de investigación se desarrolla en el comercio internacional donde confluyen flujos de importaciones, exportaciones y balanza comercial del sector productivo de los países miembros de ambas regiones. De modo que en esta investigación se identifica como problema la poca vinculación comercial entre ambas regiones. No obstante, ambos mecanismos tienen similitudes en su integración económica y comercial; una de estas es que las dos regiones están formadas por economías en desarrollo con objetivos similares como el libre tránsito de bienes, servicios, capitales y personas (Pérez y Uribe, 2017).

\section{Antecedentes AP y ANSEA}

En primer lugar, la AP y la ANSEA son dos procesos de integración económica de países emergentes y con vecinos poderosos. En el caso de la Asociación, colinda al norte con China, Corea del Sur y Japón; la Alianza hace frontera con Estados Unidos y Canadá.

Dichos procesos se han desarrollado en diferentes etapas y han tenido momentos históricos y políticos en los cuales tuvieron períodos de economía cerrada, así como de apertura. Sin embargo, existen algunas similitudes, como la que muestra este artículo, además de sus interacciones con otras economías, con los países vecinos, con organismos internacionales y otros.

La AP se integró tras la declaración de Lima de 2011 y se constituyó formalmente el 6 de junio del 2012, la cual, además de México, está formada por Colombia, Chile y Perú. Dicha asociación se creó para articular la política, la economía, los negocios, la educación, el medio ambiente y otros temas de cooperación. "El objetivo fundamental de esta alianza es establecer un bloque de integración que promueva el crecimiento, desarrollo y competitividad de sus miembros" (Ministerio de comercio exterior y turismo de Colombia - MINCIT, 2015).

Es posible darse cuenta de su gran potencial, ya que, de acuerdo con Alianza del Pacífico (2018), "dicha alianza reúne cuatro de las principales economías del continente. Una población superior a 214 millones de personas, equivalente al $36 \%$ del total de la población de América 
Latina y del Caribe. Se trata de economías abiertas muy dinámicas" (Alianza del Pacífico, 2018). Hoy, como bloque, la AP concentra el $39 \%$ del PIB de América Latina y el Caribe, además de ser la $8^{\mathrm{a}}$ potencia exportadora del mundo. Adicionalmente, como lo comenta el diario Excélsior, la Alianza del Pacífico ha logrado inspirar a líderes, ciudadanos, empresarios y gobiernos para hacer realidad el anhelo histórico de integración latinoamericana, con un éxito que ha rebasado cualquier expectativa (Banco Interamericano de Desarrollo - BID, 2016).

La integración económica y comercial dentro de la AP no sigue el modelo tradicional. Ese es uno de los Acuerdos de Integración Profunda que avanza en lo que se puede, pero en lo técnico se tienen tratados de libre comercio bilaterales entre todos los miembros, entendiendo que el comercio se liberará con base en las posibilidades de cada país. En los temas en que se pueden avanzar se hacen acuerdos o declaraciones en las reuniones anuales de la Alianza (Alianza del Pacífico, 2014). La desgravación de AP es del $92 \%$ de las partidas arancelarias y desgravación paulatina del $8 \%$ restante, además de compromisos generales en temas de obstáculos técnicos al comercio, medidas fitosanitarias, inversión, comercio electrónico, etc. (SELA, 2014).

Así bien, Pérez y Uribe (2018) destacan que la AP cuenta con la integración de sus bolsas de valores a través del Mercado Integrado Latinoamericano - MILA, el Consejo Empresarial de la Alianza del Pacífico - CEAP, la eliminación de visados, la plataforma de movilidad académica, la asistencia consular y las sedes compartidas en embajadas, entre otros (Pérez y Uribe, 2018, p. 12; Pérez y Roldán, 2015).

Actualmente, la AP cuenta con 57 países observadores, ha realizado 12 cumbres presidenciales y alcanzó la categoría Estado Asociado que le permite negociaciones en bloque con terceros. Al mismo tiempo, a ella desean integrarse países como Australia, Canadá, Nueva Zelanda y Singapur en la condición de Estado Asociado.

\section{ANSEA}

Por otro lado, se encuentra la ANSEA que fue creada en 1967 e integrada por diez naciones. Los países fundadores fueron Filipinas, Indonesia, Malasia, Tailandia y Singapur. La intención de fundar esta asociación era mantener una región pacífica y libre de armas nucleares en el contexto histórico de la Guerra Fría que se estaba viviendo en la región, principalmente en Vietnam que no formó parte sino hasta 1995. Brunei Darussalam se incorporó en 1984; Laos y Myanmar entraron en 1997 y el último país en integrarse fue Camboya en 1999 (ANSEA, 2018).
En 1992 en la $4^{\text {a }}$ cumbre se decidió la creación del Área de Libre Comercio de ANSEA, conocido como The ASEAN Free Trade Area - AFTA por sus siglas en inglés, la cual entraría en vigor hasta 2002 y que surgió como respuesta a la creación de bloques comerciales como el Tratado México Estados Unidos y Canadá T-MEC, Unión Europea, Mercosur, así como a la apertura comercial de China (ANSEA, 2018).

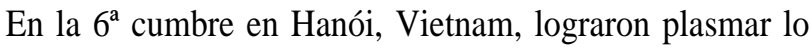
que se conoció como Visión 2020, en la cual se invitaba a la unidad de ANSEA en la dinámica del desarrollo para forjar una integración económica de la región. Se pretendió crear una región estable, próspera y altamente competitiva en la cual habría libre circulación de bienes, servicios, inversión, capitales y un desarrollo económico equitativo (ANSEA, 2018).

Dichas reuniones son de suma importancia ya que al principio de la creación de ANSEA, de los primeros cinco países miembros, la mayoría eran gobernados bajo una dictadura. Ejemplo de ello son los siguientes: Indonesia dirigido por Suharto, Singapur por Lee Kwan Yoo; Malasia era gobernada por el Barisan Nasional, grupo de partidos que a la fecha sigue en el poder, y Filipinas por Ferdinand Marcos. Las decisiones al centro se hacían entre ellos, a diferencia de América Latina cuyo ejemplo principal es México, quien para negociar el T-MEC tenía que consultar con todos los actores involucrados: iniciativa privada, trabajadores, partidos políticos, entre otros (El Colegio de México, 2002).

A partir de la $2^{\text {a }}$ Reunión Informal de Kuala Lumpur, Malasia (1997), se incorporaron de manera informal China, Corea del Sur y Japón, hecho que se conoce como ANSEA más 3 , y es desde entonces que estos tres países participan en todas las reuniones de la Asociación (Ramírez, 2001).

La $11^{\text {a }}$ Cumbre de Kuala Lumpur fue el marco de ANSEA más 6, ya que además de los 10 miembros de la Asociación, más los 3 mencionados en el párrafo anterior, también se sumaron Australia, India y Nueva Zelanda (Nguyen, 2016).

A lo largo de los 50 años que lleva vigente, sus objetivos se han concretado y siguen construyéndose debido a la cooperación y promoción de una cultura de colaboración económica en la que se entiende que el beneficio es directo para los países miembros. Su eslogan es "una visión, una comunidad, una identidad", el cual siguen erigiendo. El éxito de ANSEA se debe a tres factores: el espíritu que existe por la resolución rápida, adecuada y efectiva de los problemas regionales; la habilidad para adaptarse a las circunstancias cambiantes de la región y una relativa 
estabilidad económica (Hoang, 2017).

Para facilitar el comercio han implementado la armonización de normas industriales, la liberalización de inversiones en el sector de bienes, una mayor flexibilidad en el transporte aéreo, una mayor cooperación monetaria, la creación de un mercado de capitales y el libre flujo de trabajadores calificados. Cabe mencionar que el único Estado del sureste asiático que no pertenece a ANSEA es Timor Oriental debido a que se independizó en 2002, e Indonesia que lo gobernó de 1976 a 1999 no ha permitido su incorporación, aunque tampoco ha sido solicitada (Da Silva y Schroeter, 2007).

\section{Relaciones AP-ANSEA}

Existen diferencias entre ANSEA y su proceso integrador, razón por la cual más que un comparativo es una perspectiva para la AP, ya que como se ha mencionado, mientras que ANSEA cumple 52 años de creada, la Alianza tiene tan solo siete. Las diferencias han sido históricas, culturales, pero sobre todo de voluntad entre sus miembros. Una de las ventajas primordiales de ANSEA sobre AP es el consumo interno. El mercado regional representa un 24 $\%$ del comercio total en Asia, mientras que, en países como México, los productos de principal consumo son extranjeros (Granguillhome y Rubinstein, 2016).

Existe la reunión ministerial AP-ANSEA cuyos encuentros se han llevado a cabo desde el 25 de mayo de 2015 en Yakarta, Indonesia. Estos se han enfocado en la Visión Estratégica 2030 de Naciones Unidas (Sistema Económico Latinoamericano y del Caribe -SELA, 2016). Además, existe un Plan de Trabajo AP-ANSEA, vigente de 2015 a 2019, que tiene como base una amplia agenda en cuatro áreas: cooperación económica; educación y contactos persona a persona; ciencia, tecnología e innovación y desarrollo sostenible (Alianza del Pacífico, 2018). La distancia geográfica representa una serie de obstáculos, sin embargo, el principal es la falta de una estrategia conjunta entre la AP y su acercamiento al Asia Pacífico. Los intereses comerciales de la AP están enfocados al mercado norteamericano, mientras que la ANSEA realiza un comercio intrarregional y con las grandes economías asiáticas como China, Japón y Corea del Sur, como se puede observar en los mecanismos de ANSEA+3 o ANSEA+6 (Pérez y Uribe, 2017).

En ese sentido cobra importancia este estudio para identificar el potencial de complementariedad económica en bienes que pueden tener los dos bloques. Esto permitirá identificar concordancias para propiciar una cooperación económica más profunda y brindar elementos para un acercamiento estratégico interregional (Pérez y Uribe,
2017).

Como se puede observar, ya existe un mecanismo de contacto entre la AP y ANSEA, en el que para efectos de este estudio sobresale la cooperación económica y buenas prácticas comerciales. Teniendo en cuenta lo anterior, la hipótesis de este artículo se plantea de acuerdo con cada una de las dimensiones que son exportaciones, importaciones y balanza comercial.

Hipótesis 1. Existen diferencias significativas entre el crecimiento de las exportaciones de AP y crecimiento de las exportaciones de ANSEA.

Hipótesis 2. Existen diferencias significativas entre el crecimiento de las importaciones de AP y el crecimiento de las importaciones de ANSEA.

Hipótesis 3. Existen diferencias significativas entre la media de crecimiento de la balanza comercial de AP y la media de crecimiento de la balanza comercial de ANSEA.

\section{METODOLOGÍA}

\section{Diseño}

Las variables estudiadas fueron las exportaciones, las importaciones y la balanza comercial. Se analizaron a través de Statistical Package for the Social Sciences - SPSS 22 y figuras de Excel; ambas fueron utilizadas para interpretar los datos antes mencionados de AP y ANSEA. El SPSS permitió encontrar el alfa y la correlación para poder comprobar las hipótesis. Por otro lado, el Excel facilitó los diagramas de dispersión.

"Ahora bien, en este artículo se evidencia una metodología de enfoque cuantitativo porque se evaluaron las variables de importaciones, exportaciones y balanza comercial que permitieron someter dichos datos al análisis estadístico" (Ugalde y Balbastre, 2013, p. 181).

Adicionalmente, en el presente estudio se utilizaron datos numéricos de exportación, importación y balanza comercial obtenidos de la base de datos de la Cámara de Comercio Internacional, Trademap y estadísticas del comercio para el desarrollo internacional de las empresas. Del cruce de los anteriores datos se obtuvo la información para mostrar la tendencia de 2002 a 2018.

"Asimismo, este texto tiene un alcance analítico interpretativo, ya que busca encontrar relación entre las variables de comercio internacional para llegar a un conocimiento más profundo del comercio de la AP y ANSEA" (Hurtado, 2010, p. 133). Por otro lado, se muestra 
un diseño transversal, es decir, un "estudio que no tendrá un seguimiento en el tiempo y se efectuará en un periodo determinado" (Hernández et al, 2014, p. 154), el cual abarca de 2002 a 2018, ya que aunque la AP se creó en 2012, para efectos de medición se necesitan más datos y se eligieron los años anteriores a su creación para poder realizarlos. Adicionalmente, en la ANSEA entró en vigor el AFTA a partir de 2002, con lo que se reflejaron estadísticas, porcentajes y datos relativos al crecimiento del crecimiento de dicho mercado.

Por otro lado, el artículo tiene un diseño transversal, es un "estudio que no tendrá un seguimiento en el tiempo y se efectuará en un periodo determinado" (Hernández et al, 2014, p. 154), el cual abarca de 2002 a 2018, ya que, aunque la AP se creó en 2012, para efectos de medición se necesitan más datos y se eligieron los años anteriores a su creación para poder realizarlos, además de que por otro lado, en la ANSEA entra en vigor el AFTA a partir de 2002, con lo que se reflejan estadísticas, porcentajes y datos relativos al crecimiento del crecimiento de dicho mercado.

\section{Participantes}

Este estudio utilizó estadísticas en serie de tiempo del periodo 2002 - 20181, agrupados por exportaciones totales, importaciones totales y balanza comercial total de AP y ANSEA con el mundo, tomado de Trademap. La muestra seleccionada fueron los países que integran a la Alianza del Pacífico: 1. Colombia, 2. Chile, 3. México y 4. Perú; países miembros de la ANSEA: 5. Brunei Darussalam, 6. Camboya, 7. Filipinas, 8. Indonesia, 9. Laos, 10. Malasia, 11. Myanmar, 12. Singapur, 13. Tailandia y 14. Vietnam, para un total de 14 naciones.

\section{Instrumentos}

Se realizó minería de datos a través de la información de fuentes secundarias como Trademap e informes oficiales de ANSEA y AP.

\section{Procedimiento}

Se realizó compilación de datos estadísticos de importaciones, exportaciones y balanza comercial de la base de datos Trademap, a través del uso de la técnica minería de datos. A partir de allí se tabuló la información a través de software especializado y "se expresaron los resultados a través de figuras. Finalmente se procedió a la elaboración de este artículo a la luz de los objetivos trazados, que incluye recomendaciones y conclusiones" (Lechuga et al., 2018, p. 28).

\footnotetext{
${ }^{1}$ A pesar de que AP se creó a partir del año 2012, la información estadística es seleccionada desde 2002 a 2018 con el fin de realizar el análisis estadístico en SPSS.
}

\section{RESULTADOS}

Hasta el año pasado, el comercio internacional, al igual que la actividad económica, estaba acelerándose en casi todas las regiones del mundo. La situación ha cambiado. La escalada de las tensiones comerciales entre Estados Unidos y China ha afectado el comercio mundial. Las perspectivas que enfrentan muchos países son muy duras, marcadas por una considerable incertidumbre a corto plazo (Fondo Monetario Internacional - FMI, 2019).

Para esta investigación se realizó un análisis de fiabilidad del instrumento (Hair et al., 1998) y se encontró que el 100 $\%$ de los casos eran válidos y no había valores perdidos.

Al realizar análisis descriptivos se encontró que la media de las exportaciones en países de AP es de USD $\$ 426743$ 848,06 , y la media de las exportaciones en países de ANSEA es de USD\$ 937014 009,72. Ciertamente los países de la ANSEA tienen un mayor crecimiento en las exportaciones entre $2002-2018$.

La media de las importaciones en países de AP es de USD \$421 835 425,9444, y la media de las importaciones en países de ANSEA es de USD $\$ 883249$ 561,3333. Al igual que en las exportaciones, los países de la ANSEA tienen un mayor crecimiento en las importaciones entre 2002 - 2018. La media de la balanza comercial en países de AP es de USD \$ 4908 422,11 y la media de la balanza comercial en países de ANSEA es de USD \$53 764 448,38. Nuevamente se observa que los países de la ANSEA tienen un mayor crecimiento en las importaciones entre 2002 - 2018.

A continuación se realiza un análisis de normalidad. Para Quezada (2017), la medida Keiser Meyer Olkin - KMO permite comparar la magnitud entre los componentes de la variable. El estadístico varía entre 0 y 1 .

H0: KMO 0: Modelo Factorial es inadecuado

H1: KMO 1: Modelo Factorial es adecuado (KMO > $0,50)$.

El resultado de este ejemplo es de $\mathrm{KMO}=0,603$, por lo que se acepta que el modelo factorial es adecuado para el análisis de los componentes de la variable imagen. Como $n$ es mayor que 30, se utilizó la prueba de KolmogorovSmirnov; entonces, el P-Valor es $>/=\alpha$. Los datos provienen de una distribución normal. Las variables exportaciones, importaciones y balanza comercial se 
comportan normalmente.

A partir de los resultados obtenidos en la prueba T Student se postula que el $\mathrm{P}$ valor es $<\alpha$ y se acepta la hipótesis alternativa; es decir que existen diferencias significativas entre las varianzas, excepto en la balanza comercial. Existen diferencias significativas entre la media del crecimiento de las exportaciones de AP y la media del crecimiento de las exportaciones de ANSEA. Existen diferencias significativas entre la media del crecimiento de las importaciones de AP y la media del crecimiento de las importaciones de ANSEA. Las diferencias entre la media del crecimiento de la balanza comercial de AP y la media del crecimiento de la balanza comercial de ANSEA no tienen diferencias significativas. Las categorías analizadas fueron: exportaciones, importaciones y balanza comercial.

Las exportaciones de AP en 2018 fueron de USD\$ 615
006,333 mientras que las de ANSEA fueron USD\$ 1255 546,867 . Como se observa en la figura 1 , la variable exportaciones sigue una relación lineal, ya que tiende a una línea recta ascendente. Ambos grupos de países muestran la misma tendencia, aunque con diferencias en el tamaño de sus exportaciones: las de ANSEA son casi el doble de las de AP, lo cual se puede explicar por el número de países que la integran, que son 10 en comparación de los cuatro pertenecientes a AP.

En este sentido, el crecimiento de las relaciones comerciales de AP con Asia - Pacífico impulsa las exportaciones desde su creación, lo cual se puede observar en el crecimiento de sus exportaciones. Sin embargo, en ANSEA se observa una ligera caída entre 2017 y 2018, lo cual se puede explicar por la contracción comercial a nivel mundial de 2018 y a la guerra comercial entre Estados Unidos y China (FMI, 2019).

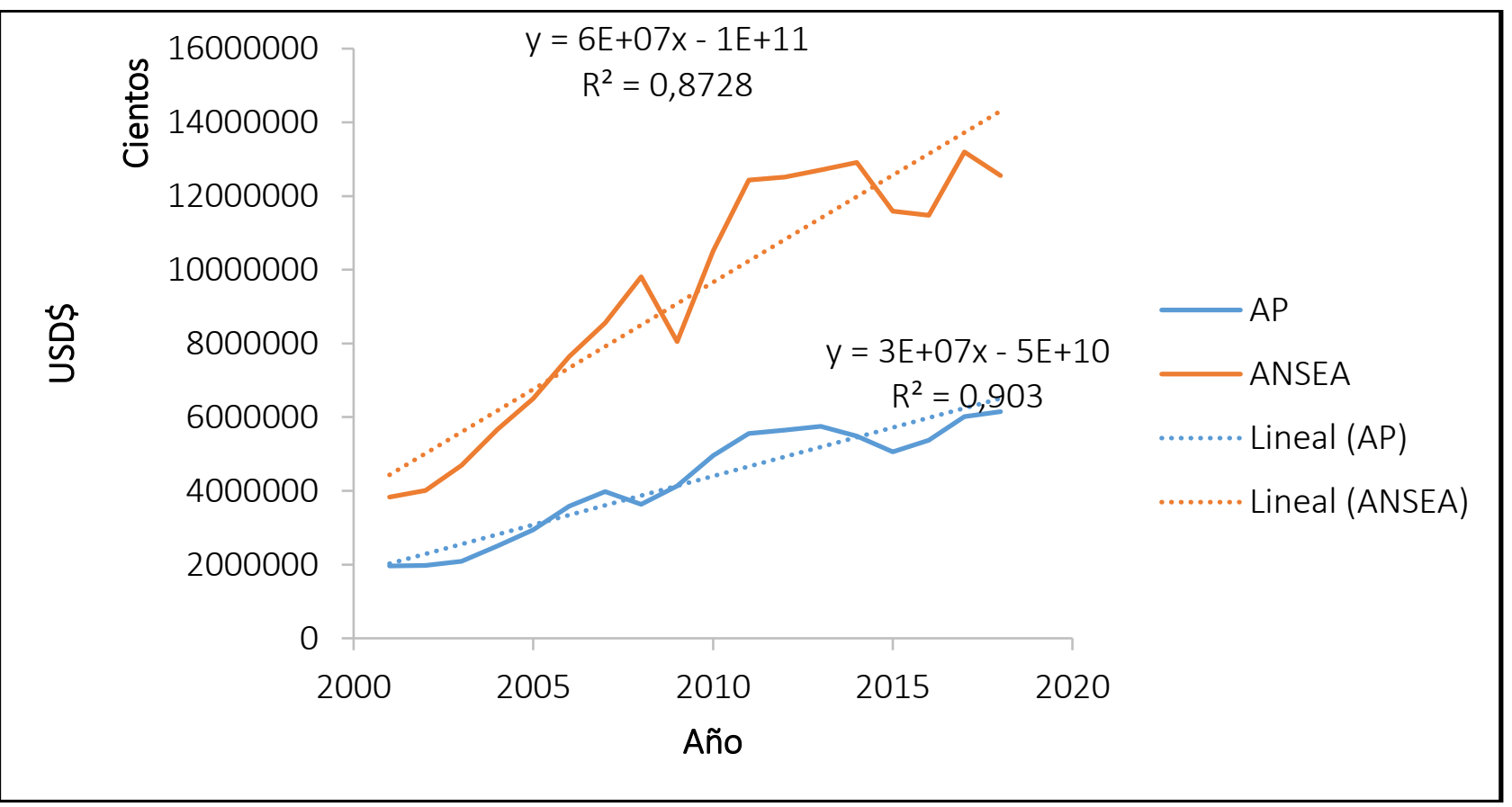

Figura 1. Correlación Exportaciones AP-ANSEA 2001- 2018

Fuente: elaboración propia basada en los datos Trademap (2019).

Como se observa en la figura 2, las importaciones de AP en 2018 fueron de USD\$ 632 832,944, mientras que las de ANSEA fueron USD $\$ 1429 \quad 159,576 . \mathrm{La}$ variable importaciones sigue una relación lineal que tiende a una línea recta ascendente. Al igual que en las exportaciones ambos grupos de países muestran la misma tendencia, aunque con diferencias en el tamaño de sus importaciones. $\mathrm{Al}$ igual que las exportaciones, las de ANSEA son un poco más del doble de las de AP, lo cual se puede explicar por el número de países que la integran, como en las exportaciones. De modo que la creciente demanda mundial de bienes y servicios impulsa las importaciones de AP y ANSEA, que a diferencia de sus exportaciones siguen creciendo en el último año y no presentan disminución como sí lo sufrieron sus exportaciones. 


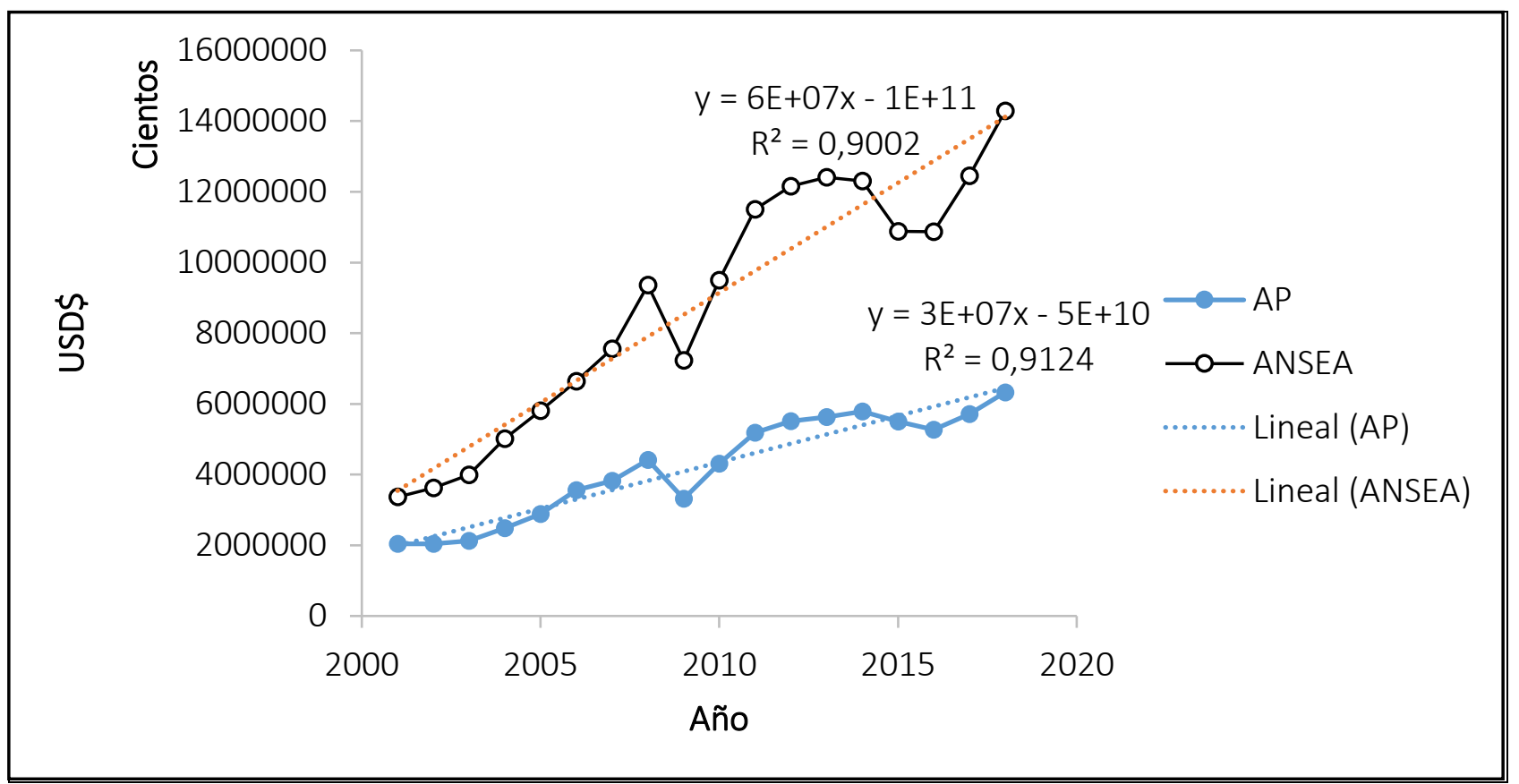

Figura 2. Correlación Importaciones AP-ANSEA 2001- 2018

Fuente: elaboración propia basada en los datos Trademap (2019).

Corresponde ahora el análisis de la balanza comercial. Se observa que la balanza comercial para AP se mantiene con crecimientos y decrecimientos, pero en una línea horizontal; mientras que la ANSEA parecería ir a la baja, tal como se observa en la figura 3. Es decir, la ANSEA presenta un déficit comercial, así como lo indica el último año revisado 2018, el cual fue de un valor de USD\$-173 612,709 , mientras que las anteriores del mismo ANSEA mostraron superávit.

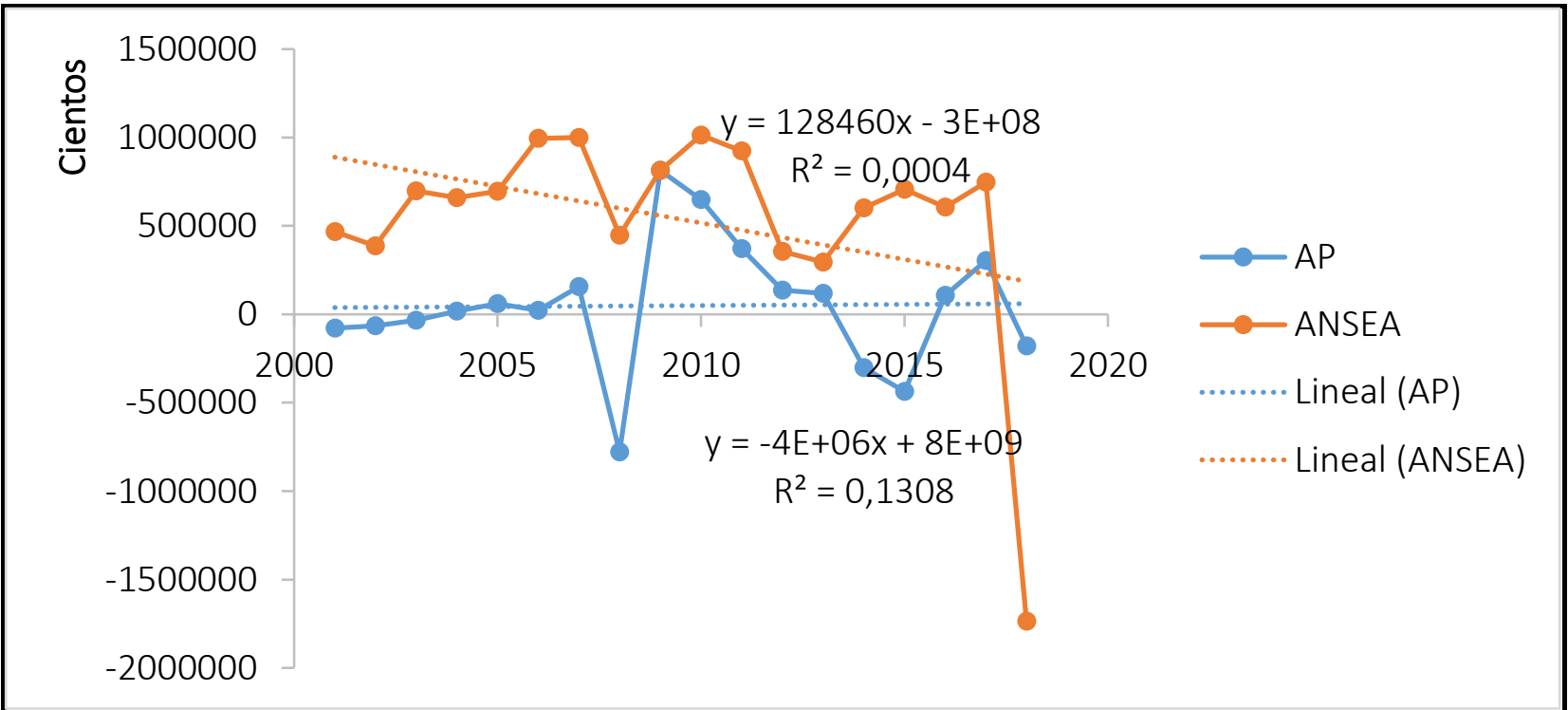

Figura 3. Correlación Balanza Comercial AP-ANSEA 2001- 2018

Fuente: elaboración propia basada en los datos Trademap (2019).

Ahora bien, la AP, aunque muestra una línea horizontal en

el mismo periodo, también tuvo déficit comercial en 2018 
de USD\$ -17 826,611. Mucho menor al de ANSEA, pero eso es debido al tamaño de su comercio. $\mathrm{Y}$ al igual que ANSEA, los años 2017 y 2016 muestran superávit comercial. La diferencia de ANSEA es que desde 2002 el único déficit comercial que sufrió fue en 2018.

Otra prueba que se hizo fue la de correlación: dos variables se correlacionan o están relacionadas cuando ambas covarían, es decir, varían de forma conjunta. Dicha variación se advierte en los diagramas de dispersión anteriores y para determinar la magnitud de la covariación se dispone de índices, denominados coeficientes de correlación. Para las variables cuantitativas existen dos índices que permiten cuantificar la relación: la covarianza y el coeficiente de correlación de Pearson. (Landero y González, 2016, p. 338).

En este sentido, para el tema abordado en este artículo las variables tratadas y las hipótesis hechas son confirmadas tanto para las exportaciones como para las importaciones en las cuales, tal como se comentó anteriormente, son mayores para ANSEA. Lo que cambia es la balanza comercial en la cual ANSEA desde 2002 ha tenido superávit, excepto para 2018 cuando muestra un déficit comercial. La correlación reafirma las hipótesis y muestra la divergencia en la balanza comercial.

Por otro lado, la regresión es una técnica estadística que analiza simultáneamente más de dos variables con el propósito de especificar y estimar una variable de interés comúnmente llamada endógena, "la cual es explicada por un conjunto de variables explicativas llamadas exógenas, con el objetivo de estudiar las relaciones existentes entre las variables" (Quezada, 2017, p. 214). La prueba estadística de regresión lineal se realiza para comprobar si existe una relación lineal entre dos variables: una de manera dependiente y otra independiente. El valor de $\mathrm{R}$ (Regresión) nos indica un número 1, "lo cual indica que el modelo es óptimo para la realización de prueba y el coeficiente de determinación (R2) es de $100 \%$. Este último valor muestra que el modelo es óptimo para la prueba" (Quezada, 2017, p.224).

\section{DISCUSIÓN}

A la luz de los objetivos trazados, en el presente artículo se encuentra que los bloques económicos objeto de análisis de este estudio han experimentado un crecimiento sostenido de sus exportaciones e importaciones. No obstante, las exportaciones en la ANSEA son superiores a las de AP, lo que se explica por el número de países que lo integran: mientras en la ANSEA son 10, en la AP son cuatro. En este sentido, como bien apunta la Secretaría Permanente del SELA (2016), existen diferencias en el tratamiento de los distintos procesos de integración subregionales que pueden llevarse en el mundo. Así, existen diferentes etapas de integración económica, como lo expuso Petit (2014), y aunque se han utilizado diferentes indicadores cuantitativos para la medición de la integración económica en el mundo no ha sido aceptado por la academia un criterio único, dada la multidimensionalidad de los distintos procesos de integración.

En contraste, el comportamiento de la balanza comercial difiere en la medida en que en la AP existe déficit comercial y en la ANSEA solo se presenta en el último periodo de análisis. Esto respalda la teoría económica de los beneficios del intercambio comercial, como sustenta Villagra et al (2018) "la integración económica y su consiguiente apertura comercial beneficia a los miembros al permitirles el acceso a mercados más grandes y expandir con ello su aparato exportador incrementando su crecimiento potencial compatible con la balanza de pagos" (p.50).

Aquí cabe resaltar que esta divergencia con la teoría se explica en este periodo debido a factores externos como la guerra comercial entre Estados Unidos y China que repercute en todo el mundo y en particular en la ANSEA, aunque la explicación más clara dada por el ISEAS es que dicho déficit es debido al comercio con China, país del cual se está importando más de lo que se exporta (Basu, 2018). La mayoría de los países se encuentran en dicha posición. Un ejemplo es México, el cual en algunos años ha tenido casi alrededor del $300 \%$ de déficit y que la misma China ha buscado subsanar. Estados Unidos es el país que ha manifestado su malestar ante dicha situación y en el último año se han confrontado ambos países en lo que se ha llamado "guerra comercial".

Con relación al comercio entre ANSEA y China, la Asociación ha gozado de superávit en la mayor parte del tiempo y no es sino hasta 2018 que tuvo un déficit de \$243 mil millones de dólares, lo cual implica que China importa más servicios del mundo comparado con ANSEA. Pero, bilateralmente, China exporta ingeniería y manufacturas a ANSEA, e importa transporte, servicios financieros y de construcción.

En síntesis, el comercio internacional es un aspecto crucial para ambos procesos de integración, ya que contribuye al crecimiento económico y a fortalecer los lazos económicos entre las naciones que integran dichos procesos. En su estudio, Osorio (2013) observa los efectos positivos en los 
intercambios comerciales de los procesos de integración económica, así como el hecho de que una mayor integración económica, entre otros factores, ha tenido una influencia positiva en el crecimiento económico de los países que gozan de integración regional. Sin embargo, existen preocupaciones por los cambios en las posiciones de las economías como Estados Unidos y China, para continuar con la situación global de libre comercio que ha prevalecido en los últimos años.

\section{CONCLUSIÓN}

En la primera hipótesis se aseveró que existen diferencias significativas entre el crecimiento de las exportaciones de AP y el crecimiento de las exportaciones de ANSEA. Las pruebas y los resultados confirman esta hipótesis. Como se menciona en el apartado de exportaciones, la razón de esto puede ser el que son 10 países en la Asociación y cuatro en $\mathrm{AP}$, por lo que el número de países miembros y el dinamismo de la economía asiática han impulsado el monto de las exportaciones de ANSEA. En la hipótesis 2, que se refiere a las diferencias significativas entre el crecimiento de las importaciones de AP y el crecimiento de la misma de ANSEA, sucede lo mismo que en la primera. Nuevamente, las pruebas y los resultados lo indican.

Por último, la tercera hipótesis es en relación con las diferencias significativas a la media de crecimiento de la balanza comercial de AP y la media de crecimiento de la balanza comercial de ANSEA. Es en este indicador en donde no se confirma, debido a que hay un descenso para la Asociación en 2018; por primera vez desde 2002 existe un déficit en ANSEA. El motivo, como se ha comentado en ese apartado, puede ser la confrontación comercial entre China y Estados Unidos que ha afectado a la región. La AP sigue con la misma tendencia lineal $\mathrm{y}$, aunque ha tenido varios déficits en los periodos analizados, en la gráfica no se observa esa caída, a diferencia de ANSEA que sí la muestra.

El comercio exterior interregional es un mecanismo que podría servir como catalizador para el desarrollo económico de la Alianza del Pacífico y ANSEA. No obstante, los flujos comerciales entre ambas regiones son aún muy bajos. Resulta necesario explorar mecanismos que incentiven al tejido empresarial de ANSEA a hacer negocios en la Alianza del Pacífico y, a través de esta, poder proyectarse hacia otros países de América Latina o incluso el Tratado entre México, Estados Unidos y Canadá -T-MEC.

La AP quiere dar un énfasis especial a sus relaciones con Asia - Pacífico. Con lo observado en este estudio vemos que ambos mercados se han comportado de forma similar, sin embargo, deben vincularse ambos mercados y en particular la AP. Observar la forma en que se relaciona la ANSEA con sus vecinos y con las potencias mundiales quizás podría ser útil en plena confrontación comercial entre los Estados Unidos y China.

\section{Declaración sobre conflictos de interés}

Las ideas, opiniones, comentarios y conclusiones expresadas en este documento son responsabilidad de los autores y son independientes de su institución de afiliación. Adicionalmente, durante la realización del trabajo y la redacción de este documento, no se ha incurrido en ningún conflicto de interés.

\section{REFERENCIAS BIBLIOGRÁFICAS}

Alianza del Pacífico. (2018). Alianza del Pacifico.

https://alianzapacifico.net

Alianza del Pacífico. (2015). Abecé.

https://alianzapacifico.net/wp-

content/uploads/2015/06/abc_AP.pdf

Alianza del Pacífico (2014). Protocolo adicional al acuerdo marco de la Alianza del Pacifico. https://alianzapacifico.net/download/protoc olo-adicional-al-acuerdo-marco-de-laalianza-del-pacifico/

Banco Interamericano de Desarrollo - BID. (2016).

Integración económica regional: ¿qué es la Alianza del Pacífico y hacia dónde va? https://www.youtube.com/watch?v=7XclRb a541Q

Basu, D. S. (2018). RCEP in 2018? - Expectations must be tempered. ISEAS. https://www.iseas.edu.sg/medias/commenta ries/item/6995-rcep-in-2018-expectationsmust-be-tempered-by-sanchita-basu-das

Da Silva, K. C. y Schroeter, S. D. (2007). TimorLeste por trás do palco. Cooperacao internacional dialética da formacao do Estado (1a ed.). Editora UFMG. https://books.google.com.mx/books?id=lFxl 
$\underline{C}$ jJIaNIC\&printsec $=$ frontcover $\& h \mathrm{l}=\mathrm{es} \&$ sou

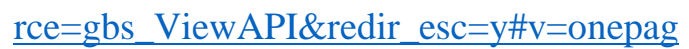

$\underline{\mathrm{e} \& \mathrm{q} \& \mathrm{f}=\text { false }}$

El Colegio de México, Centro de Estudios de Asia y África (2002). Asia Pacífico Programa de Estudios APEC.

Fondo Monetario Internacional - FMI. (2019). Perspectivas de la Economía Mundial - Abril de 2019.

https://www.imf.org/es/Publications/WEO/I $\underline{\text { ssues/2019/03/28/world-economic-outlook- }}$ april-2019

Granguillhome, R. y Rubinstein, M. (2016). México y la ANSEA: en busca de una relación estratégica. Revista Mexicana de Política Exterior, $\quad 108, \quad 175-193$. https://revistadigital.sre.gob.mx/images/stor ies/numeros/n108/granguillhomerubinstein. pdf

Hair, J., Anderson, R., Tathan, R. y Black, W. (1998). Análisis multivariante (5 $5^{a}$ Ed.). Pearson.

Hernández, R., Fernández, C. y Baptista, P. (2014). Metodología de la investigación. Mc Graw Hill.

Hoang, T. H. (2017). Finding Common Ground to Move. ASEAN Focus (6), 2-3. https://www.iseas.edu.sg/images/pdf/NOV_ ASEANFOCUS_WEB.pdf

Hurtado, J. (2010). Guía para la comprensión holística de la ciencia. Fundación Sypal.

Landero, H. R. y González, R. M. T. (2016). Estadística con SPSS y metodología de la investigación. Trillas.

Lechuga, J., Cazallo, A., Meñaca, I., Martínez, H., García, J., Olivero, E. y García, J. (2018).
Análisis del comportamiento de las exportaciones del Departamento del Atlántico (Colombia) entre 2012 - 2016. Revista Espacios, 39(22), 22 - 41. https://www.revistaespacios.com/a18v39n2 2/a18v39n22p22.pdf

Ministerio de comercio exterior y turismo de Colombia - MINCIT. (2015). Ministerio de comercio exterior y turismo. http://www.mincit.gov.co/tlc/publicaciones. php?id=7180Ministerio

Nguyen, T. (2016). Economic Integration in ASEAN+3: A Network Analysis. Journal of Economic, 31(2), 275-325. https://www.jstor.org/stable/43783268?seq= $\underline{1}$

Osorio, C. M. I. (2013). Integración comercial y crecimiento económico en América Latina (tesis doctoral). Universidad Autónoma de Barcelona, Barcelona (España). https://www.tesisenred.net/bitstream/handle /10803/123196/mioc1de1.pdf?sequence=1

Pérez, R. C. A. y Uribe, J. M. T. (2017). Alianza del Pacífico y ASEAN: hacia una cooperación económica más profunda. ALADAA.

Pérez, R. C. y Uribe, J. M. T. (2018). La Alianza del Pacífico en los nuevos escenarios de integración Asia-Pacífico (1a ed.). EAFIT.

Petit, P. J. G. (2014). La teoría económica de la integración y sus principios fundamentales. Revista Venezolana de Análisis de Coyuntura. 20(1), 137-162. https://www.redalyc.org/pdf/364/36433515 007.pdf

Quezada, L. N. (2017). Estadística con SPSS 24. (1 ${ }^{a}$ 
COMERCIO INTERNACIONAL ENTRE ALIANZA DEL PACÍFICO Y ANSEA: UN ANÁLISIS COMPARATIVO

ed). $\quad$ Editorial $\quad$ Macro.

https://editorialmacro.com/catalogo/estadisti ca-con-spss-24/

Ramírez, L. B. V. (2001). ANSEA más 3: un proyecto de integración regional en Asia del Pacífico (Tesis de pregrado, ITAM). Archivo digital. https://relacionesinternacionales.itam.mx/es/1/pagin as/nuestros-egresados-3

Ramírez, B. J. J. (16 de Octubre de 2018). Entrevista.

(F. R. González Ayala, Entrevistador) México, CDMX, México.

Pérez, C. y Roldán, A. (2015). Is the Pacific Alliance a potential pathway to the Free Trade Area of the Asia-Pacific? Philippines Journal of Development, 41-42(1-2a), 1-20. https://econpapers.repec.org/paper/phdpjdev t/pjd_5f2014-2015_5fvol_5f_5f41-

42_5fnos_5f_5f1-2a.htm

Sistema Económico Latinoamericano y del Caribe SELA (2016). Índice de Integración de América Latina y el Caribe. http://www.sela.org/media/3204627/dt-2-

indice-de-integracion-definitivo.pdf

Sistema Económico Latinoamericano y del Caribe SELA (2014). Evolución de la Alianza del Pacífico. http://pacificallianceblog.com/wpcontent/uploads/2018/01/2014-SELAEvolucion-de-la-Alianza-del-Pacifico-1.pdf

Ugalde, B. N. y Balbastre-Benavent, F. (2013). Investigación cuantitativa e investigación cualitativa: buscando las ventajas de las diferentes metodologías de investigación. Revista de Ciencias económicas, 31(2), 179187.

https://revistas.ucr.ac.cr/index.php/economi cas/article/view/12730

Villagra-Piña, A., Mendoza-González, M. y Quintana-Romero, L. (2018). Un enfoque comparativo sobre la integración y apertura comercial en el crecimiento económico de la Unión Europea y América Latina. Revista Mexicana de Economía y Finanzas, 13(1), 27-52.

https://doi.org/10.21919/remef.v13i1.258 